\title{
Application value of laparoscopy in radical mastectomy and omental breast reconstruction
}

\author{
GUANGTAI SHEN and XIAOQIANG YU \\ Department of Breast Surgery, Inner Mongolia Xing'an League People's Hospital, \\ Ulanhot, Inner Mongolia 137400, P.R. China
}

Received January 22, 2019; Accepted May 3, 2019

DOI: $10.3892 / \mathrm{ol} .2019 .10339$

\begin{abstract}
Application value of laparoscopy in radical mastectomy and omental breast reconstruction was explored. The medical records of 104 patients undergoing radical mastectomy and omental breast reconstruction from July 2015 to August 2017 in Inner Mongolia Xing'an League People's Hospital were retrospectively analyzed. Fifty-three patients undergoing laparoscopic surgery were the experimental group and 51 patients undergoing open surgery were the control group. The surgery conditions, upper limb function, postoperative complications, volume similarity between unilateral subcutaneous glands and autologous omentum, cosmetic excellent rate and treatment satisfaction were observed. The surgical duration of the patients in the experimental group was significantly longer than that in the control group, but the intraoperative blood loss was less and hospital stay was significantly shorter than that in the control group $(\mathrm{P}<0.05)$. The improvement of upper limb function in the experimental group was significantly better than that in the control group $(\mathrm{P}<0.05)$. The cosmetic excellent rate in the experimental group was significantly higher than that in the control group $(\mathrm{P}<0.001)$. The incidence of postoperative complications in the experimental group was significantly less than that in the control group $(\mathrm{P}<0.001)$. The satisfaction degree of patients in the experimental group was significantly higher than that in the control group $(\mathrm{P}<0.001)$. Filling the autologous omentum while excising the unilateral mammary gland is an equivalent replacement, which can make the appearance of the breast on the operation side similar to that on the healthy side, thus improving the excellent rate of breast beauty and patient satisfaction.
\end{abstract}

\section{Introduction}

As a malignant tumor, breast cancer is highly prevalent in females and seriously affects their physical and mental

Correspondence to: Dr Xiaoqiang Yu, Department of Breast Surgery, Inner Mongolia Xing'an League People's Hospital, 12 Hanshan East Street, Ulanhot, Inner Mongolia 137400, P.R. China E-mail: bq969m@163.com; 977813293@qq.com

Key words: radical mastectomy, laparoscopy, omental breast reconstruction, application value health (1). It has long been treated by traditional total mastectomy that has a high success rate and low local recurrence rate $(2,3)$. However, severe asymmetry caused by postoperative absence of unilateral or bilateral breasts leads to physical and psychological disorders, which may seriously affect the physical and mental health of the patient (4). The greater omental tissue is soft and muscle-free, which is close to the breast cancer tissue and does not cause disuse atrophy, so it is the ideal breast reconstruction filler for female patients after radical mastectomy (5). The first application of greater omentum for breast reconstruction after radical mastectomy was in 1963, which was not promoted because open wounds for the extraction of the greater omentum resulted in a large trauma to the patient (6). However, according to a study in 2002 , laparoscopy was used to obtain the greater omentum in order to fill the breasts, which effectively reduced the trauma and was widely recognized (7).

The traditional radical mastectomy is an open surgery, which causes a great trauma to the patient and is not conducive to postoperative recovery. Additionally, papillary necrosis, incisional scar proliferation and slow gastrointestinal motility and other postoperative complications have been difficult problems in clinical practice $(8,9)$. With the development of medical technology, laparoscopy has gradually been applied in breast diseases due to its simple operation and small trauma (10). Currently, there are few studies on the application of laparoscopy in radical mastectomy and omental breast reconstruction, the applied value of which is therefore evaluated in this study.

\section{Patients and methods}

General information. A retrospective analysis was made on the data from 104 patients receiving radical mastectomy and omental breast reconstruction from July 2015 to August 2017 in Inner Mongolia Xing'an League People's Hospital (Ulanhot, China). The patients were divided into two groups: 51 patients in the control group, who had open surgery of radical mastectomy and omental breast reconstruction was performed, and 53 patients in the experimental group who had radical mastectomy and omental breast reconstruction was performed assisted by a laparoscope. The two groups of patients were comparable since they were not significantly different in terms of age, BMI and clinical stage $(\mathrm{P}>0.05)$ (Table I). 
Table I. General information.

\begin{tabular}{|c|c|c|c|c|}
\hline Factors & Experimental group $(\mathrm{n}=53)$ & Control group $(\mathrm{n}=51)$ & $\mathrm{t} / \chi^{2}$ value & P-value \\
\hline Age & & & 0.000 & 0.979 \\
\hline$\leq 40$ & $30(56.60)$ & $29(56.86)$ & & \\
\hline$>40$ & $23(43.40)$ & $22(43.14)$ & & \\
\hline $\operatorname{BMI}\left(\mathrm{kg} / \mathrm{m}^{2}\right)$ & & & 0.003 & 0.960 \\
\hline$\leq 21$ & $20(37.74)$ & $19(37.25)$ & & \\
\hline$>21$ & $33(62.26)$ & $32(62.75)$ & & \\
\hline Marital status & & & 0.174 & 0.895 \\
\hline Married & $41(77.36)$ & $40(78.43)$ & & \\
\hline Unmarried & $12(22.64)$ & $11(21.57)$ & & \\
\hline Pathological type & & & 0.203 & 0.977 \\
\hline Papillary carcinoma & $15(28.30)$ & $14(27.45)$ & & \\
\hline Medullary carcinoma & $13(24.53)$ & $13(25.49)$ & & \\
\hline Mucinous adenocarcinoma & $12(22.64)$ & $13(25.49)$ & & \\
\hline Adenoid cystic carcinoma & $13(24.53)$ & $11(21.57)$ & & \\
\hline Tumor diameter (mm) & $18.75 \pm 2.96$ & $18.65 \pm 2.81$ & 0.177 & 0.860 \\
\hline Tumor stage & & & 0.049 & 0.826 \\
\hline Stage I & $24(45.28)$ & $22(43.14)$ & & \\
\hline Stage II & $29(54.72)$ & $29(56.86)$ & & \\
\hline Childbearing history & & & 0.014 & 0.905 \\
\hline Yes & $40(75.47)$ & $39(76.47)$ & & \\
\hline No & $13(24.53)$ & $12(23.53)$ & & \\
\hline
\end{tabular}

Inclusion and exclusion criteria. Inclusion criteria were: Patients with pathological diagnosis of breast cancer; patients with single tumor and with a maximum diameter of $3 \mathrm{~cm}$; patients aged over 18 years; patients with surgical indications. Exclusion criteria were: Patients with severe liver and kidney dysfunction; patients with a predicted survival time of less than 1 year; patients unsuitable for surgery; patients with distant metastases of tumor; patients with a history of radiotherapy or chemotherapy.

This study was carried out with the approval of the Ethics Committee of Inner Mongolia Xing'an League People's Hospital and an informed consent from all the patients and their families.

Surgical methods. General anesthesia was performed in both groups. The specific surgical methods of the experimental group were the following: Holes were separately made on the tumor side at the patient's axillary, the outer edge of the breast, the outer surface of the areola, and the inframammary fold as the laparoscopic operation holes, and the lymph nodes of the axillary was cleaned through the three holes at the outer surface of the areola, the outer edge of the breast, and the inframammary fold. The mammary gland was removed through the holes at the axillary, the inframammary fold, and the outer edge of the breast. An extension of $4 \mathrm{~cm}$ was made at the inframammary fold incision, through which the mammary gland and the axillary lymph nodes were taken out, and two drainage tubes were separately connected to the incision of inframammary fold and the remaining cavity of mammary gland. Three operation holes were made respectively at the lower part of the right costal margin, and at the right and left locations $7 \mathrm{~cm}$ away from the umbilicus, and an observation hole in the abdomen was established to observe the size and adhesion of the omentum. Next, an ultrasonic scalpel was used to separate the omentum on the transverse colon with every possible effort to retain all three branches of the omentum. A $25 \mathrm{~cm}$ longitudinal tunnel was then prepared below the xiphoid to get subcutaneous connection with the extended incision of the inframammary fold, and the separated omentum was dragged into the breast through the tunnel. After confirming a good beating of the branches of the omentum and a satisfactory breast filling, the extended incision was sutured and all the puncture holes were closed. Finally, the bandaging was carried out without pressing the breast.

The specific surgical methods of the control group were the following: A $25 \mathrm{~cm}$ incision on the tumor side from the axillary to the inframammary fold was finished to clean and remove the lymph nodes and mammary gland. The omentum was separated through an incision $7 \mathrm{~cm}$ away from the upper abdomen, then the same steps as in the tunnel establishment and breast filling in the experimental group were performed.

Observation indicators. i) The operation time, blood loss during the operation, and hospitalization time of the two groups. ii) The edema, physical feeling, and ability of abduction activity of the upper extremities within 2 months after the surgery. iii) The cosmetic effects on the treated breast were classified into four grades: excellent (with same shape as, similar color and touch feel to the healthy breast, and very 
Table II. Comparison of surgical duration, intraoperative blood loss and hospital stay in two groups.

\begin{tabular}{lccrr}
\hline Items & Experimental group $(\mathrm{n}=53)$ & Control group $(\mathrm{n}=51)$ & $\mathrm{t}$ value & P-value \\
\hline Surgical duration (min) & $112.51 \pm 16.43$ & $76.22 \pm 8.54$ & 14.05 & $<0.001$ \\
Intraoperative blood loss (ml) & $54.17 \pm 13.79$ & $102.65 \pm 18.26$ & 15.32 & $<0.001$ \\
Hospital stay (days) & $9.61 \pm 2.52$ & $14.93 \pm 3.71$ & 8.58 & $<0.001$ \\
\hline
\end{tabular}

Table III. Cosmetic results of the breast of the patients in the two groups [n (\%)].

\begin{tabular}{lcccr}
\hline Items & Experimental group $(\mathrm{n}=53)$ & Control group $(\mathrm{n}=51)$ & $\chi^{2}$ value & P-value \\
\hline Excellent & $39(73.58)$ & $11(21.57)$ & 28.17 & $<0.001$ \\
Good & $8(15.09)$ & $13(25.49)$ & 1.743 & 0.187 \\
Acceptable & $6(11.32)$ & $20(39.22)$ & 10.79 & $<0.001$ \\
Poor & 0 & $7(13.73)$ & 6.48 & $<0.050$ \\
Success rate & $47(88.68)$ & $24(47.06)$ & 20.78 & $<0.001$ \\
\hline
\end{tabular}

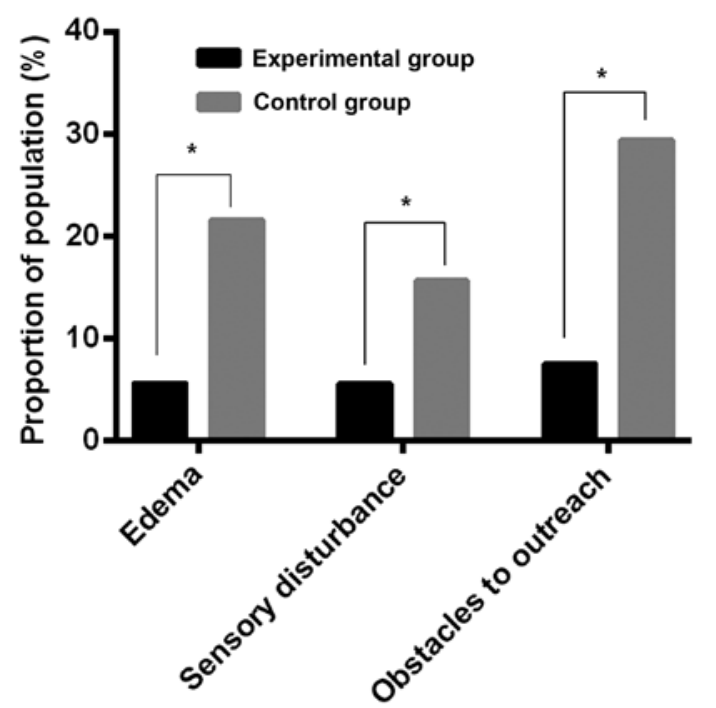

Figure 1. Comparison of upper limb function two months after operation. The ratio of the patients with edema, sensory disorder, and obstacles to outreach in upper limb function in the experimental group was significantly lower than that in the control group, and the improvement of upper limb function in the experimental group was significantly better than that in the control group, and the difference was statistically significant $\left({ }^{*} \mathrm{P}<0.05\right)$.

few surgical marks), good (with similar shape to and slightly different color and touch feel from the healthy breast), acceptable (with slight unsymmetry, different touch feel from the healthy breast, and obvious surgical marks), and poor (with substantial unsymmetry, rough hand feeling, breast atrophy or greatly changed breast shape). iv) The similarity between the volumes of unilateral subcutaneous mammary gland and autologous omentum in the two groups of patients. v) Complication during the follow-up in the two groups, including flap necrosis, subcutaneous effusion, and post-operative infection of omental transplantation. vi) Patients' satisfaction of the treatment according to the questionnaire which was classified into three grades: very satisfied, satisfied and dissatisfied. All indicators were recorded and compared between the two groups.
Statistical analysis. In this experiment, SPSS 18.0 software (Beijing ND Times Technology Co., Ltd.) was used for statistical analysis. The enumeration data were measured by Chi-square test. The measured data were expressed as mean $\pm \mathrm{SD}$. The comparison between the two groups was conducted by independent t-test. $\mathrm{P}<0.05$ indicated a statistically significant difference.

\section{Results}

Comparison of surgical duration, intraoperative blood loss and hospital stay of the patients in the two groups. The surgical duration in the experimental group was significantly longer than that in the control group, and the difference was statistically significant $(\mathrm{P}<0.05)$. However, the intraoperative blood loss and hospital stay in the experimental group were significantly better than those in the control group, and the difference was statistically significant $(\mathrm{P}<0.05)$ (Table II).

Comparison of upper limb function two months after operation. The ratio of the patients with edema, sensory disorder, and obstacles to outreach in upper limb function in the experimental group was 5.66, 5.66, and 7.55\%, respectively, which in the control group was $21.57,15.69$, and $29.41 \%$, respectively. The improvement of upper limb function in the experimental group was significantly better than that in the control group, and the difference was statistically significant $(\mathrm{P}<0.05)$ (Fig. 1).

Comparison of cosmetic results of the breast. The number of the patients whose cosmetic results were excellent, good, acceptable, and poor in the experimental group was 39, 8, 6, and 0 , respectively with a success rate of $88.68 \%$. The number in the control group was $11,13,20$, and 7 , respectively with a success rate of $47.06 \%$. The cosmetic excellent rate in the experimental group was significantly higher than that in the control group, and the difference was statistically significant $(\mathrm{P}<0.05)$ (Table III). 
Table IV. Incidence of postoperative complications.

\begin{tabular}{lccrr}
\hline Items & Experimental group $(\mathrm{n}=53)$ & Control group $(\mathrm{n}=51)$ & $\chi^{2}$ value & P-value \\
\hline Skin flap necrosis & $2(3.77)$ & $4(7.84)$ & 0.792 & 0.374 \\
Subcutaneous effusion & $1(1.89)$ & $7(13.73)$ & 5.130 & $<0.050$ \\
$\begin{array}{l}\text { Postoperative infection } \\
\text { of omental transplantation }\end{array}$ & $2(3.77)$ & $6(11.76)$ & 2.337 & 0.126 \\
Total incidence & $5(9.43)$ & $17(33.33)$ & 8.901 & $<0.050$ \\
\hline
\end{tabular}

Table V. Comparison of treatment satisfaction [n (\%)].

\begin{tabular}{lcccr}
\hline Items & Experimental group $(\mathrm{n}=53)$ & Control group $(\mathrm{n}=51)$ & $\chi^{2}$ value & P-value \\
\hline Very satisfied & $35(66.04)$ & $21(41.18)$ & 6.464 & $<0.050$ \\
Satisfied & $17(32.08)$ & $14(27.45)$ & 0.266 & 0.606 \\
Dissatisfied & $1(1.89)$ & $16(31.37)$ & 16.52 & $<0.001$ \\
Total satisfaction & $52(98.11)$ & $35(68.63)$ & 16.52 & $<0.001$ \\
\hline
\end{tabular}

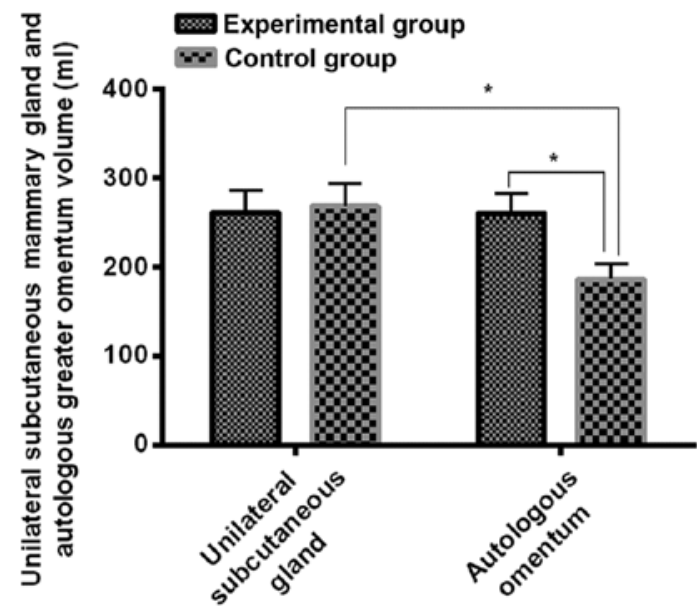

Figure 2. Comparison of the volume of unilateral subcutaneous mammary gland and autologous omentum. In the experimental group, there was no significant difference in the volumes of unilateral subcutaneous mammary gland and autologous omentum $(\mathrm{P}>0.05)$, but in the control group, the volume of autologous omentum was significantly smaller than that of unilateral subcutaneous mammary gland, and the difference was statistically significant $\left({ }^{*} \mathrm{P}<0.05\right)$.

Comparison of the volume of unilateral subcutaneous mammary gland and autologous omentum. In the experimental group, the volume of unilateral subcutaneous mammary gland and autologous omentum was respectively $261.45 \pm 24.83$ and $260.71 \pm 21.35 \mathrm{ml}$, and was respectively $268.59 \pm 25.11$ and $186.35 \pm 18.26 \mathrm{ml}$ in the control group. There was no significant difference in the unilateral subcutaneous mammary gland in the two groups $(\mathrm{P}>0.05)$. In the experimental group, there was no significant difference in the volumes of unilateral subcutaneous mammary gland and autologous omentum $(\mathrm{P}>0.05)$, but in the control group, the volume of autologous omentum was significantly smaller than that of unilateral subcutaneous mammary gland, and the difference was statistically significant $(\mathrm{P}<0.05)$ (Fig. 2).
Incidence of postoperative complications. In the experimental group, the number of the patients with skin flap necrosis, subcutaneous effusion and postoperative infection of omental transplantation was 2,1 , and 2 , respectively, with a total incidence of the complications of $9.43 \%$. The number in the control group was 4,7 , and 6 , respectively, with a total incidence of the complications of $33.33 \%$. The total incidence in the experimental group was significantly lower than that in the control group, and the difference was statistically significant $(\mathrm{P}<0.05)$ (Table IV).

Comparison of treatment satisfaction. The number of the patients who were very satisfied, satisfied and dissatisfied in the experimental group was 35,17 , and 1 , respectively, with a total satisfaction of $98.11 \%$. The number in the control group was 21,14 , and 16 , respectively, with a total satisfaction of $68.63 \%$. The total satisfaction in the experimental group was significantly higher than that in the control group, and the difference was statistically significant $(\mathrm{P}<0.05)$ (Table V).

\section{Discussion}

Surgery is the main treatment for breast cancer, but traditional surgery is not advised because of large trauma and bleeding, as well as slow recovery. Moreover, the absence of breast brings a great impact on physical and mental health of patients (11). With the development of minimally invasive technologies, laparoscopic surgery can promote the recovery of patients and reduce the incidence of complications (12).

In this study, the surgical duration in the experimental group was significantly longer than that in the control group because of complicated procedures of laparoscopic radical mastectomy and omental filling compared with the traditional surgery. However, the intraoperative blood loss and hospital stay in the experimental group were significantly better than those in the control group, indicating that laparoscopic surgery can reduce the amount of bleeding and promote the 
recovery of patients. Previous studies have demonstrated that laparoscopic surgery was effective in promoting the recovery of patients $(13,14)$. Moreover, upper limb function recovery in the experimental group was significantly better than that in the control group and complications were significantly lower than that in the control group. The study suggested that laparoscopic radical surgery and omental breast reconstruction was safer than the traditional surgery for treating breast cancer (15).

The mere use of minimally invasive techniques to remove breast cancer glands does not solve the problem of breast loss. Traditional breast conserving therapy (BCT) uses a transferred muscle flap for breast reconstruction, which not only causes large trauma, but also complications such as muscle atrophy, thus affecting the appearance of the breast (16). Some scholars put forward that omentum with similar touch feel to breast could be applied for breast filling in that omentum is rich in blood supply and can reduce the chance of skin flap necrosis, becoming the most suitable materials $(17,18)$. In radical mastectomy, the removal of unilateral subcutaneous mammary gland and the filling of residual cavity with pedicled omentum can reconstruct the breast that is similar to the original one (19). In terms of free donor, the use of endoscope in reconstruction makes the incision design under the fold of the breast. Papilla and areola is similar to the contralateral one (20). More importantly, omentum with high survival rate and flexibility can reconstruct the contralateral breast of the original type (21). The current surgical concept is that under the premise of mastering indications and ensuring the cure of lesions, do a good job of one-stage breast reconstruction, conserve the breasts as much as possible and carry out breast reconstruction if they cannot be conserved (22). According to the comparison of the unilateral subcutaneous mammary gland with the autologous omentum with respect to volume, there was no significant difference in the experimental group, but the autologous omentum was significantly smaller than the unilateral subcutaneous mammary gland in the control group. This may be due to the fact that during open surgery, the greater omentum was exposed insufficiently at both ends of the surgical field, which resulted in an insufficient cut of the greater omentum and eventually smaller reconstructed breasts. It is believed that with the help of this relationship during laparoscopy, the fully dissociative pedicled omentum can be used to reconstruct the breasts basically the same as the original breasts in terms of size, shape and hand feel. Currently, there is no relevant literature for similar reports. In this study, the treatment satisfaction in the experimental group was significantly higher than that in the control group. It is believed that the higher treatment satisfaction in the experimental group is closely related to the fast recovery, less complications and better laparoscopic breast reconstruction with greater omentum.

Compared with the traditional open surgery, laparoscopy in radical mastectomy and free omental breast reconstruction has a longer surgical duration, but it can improve the postoperative recovery and reduce the effect of surgery on upper limb function. It was also found that the greater omentum can only be fully dissociated by endoscopic surgery, and the volume of the free omentum is similar to that of unilateral mammary gland. Filling the autologous omentum while excising the unilateral mammary gland is an equivalent replacement, which can make the appearance of the breast on the operation side similar to that on the healthy side, thus improving the excellent rate of breast beauty and patient satisfaction. Therefore, laparoscopy is worth popularizing in radical mastectomy and free omental breast reconstruction.

\section{Acknowledgements}

Not applicable.

\section{Funding}

No funding was received.

\section{Availability of data and materials}

The datasets used and/or analyzed during the present study are available from the corresponding author on reasonable request.

\section{Authors' contributions}

GS wrote the manuscript, analyzed and interpreted the patient data. XY performed the experiment, designed the study and was responsible for the analysis and discussion of the data. Both authors read and approved the final manuscript.

\section{Ethics approval and consent to participate}

The study was approved by the Ethics Committee of Inner Mongolia Xing'an League People's Hospital (Ulanhot, China). Patients who participated in this study had complete clinical data. Signed written informed consent was obtained from the patients and guardians.

\section{Patient consent for publication}

Not applicable.

\section{Competing interests}

The authors declare that they have no competing interests.

\section{References}

1. Dec W: Optimizing aesthetic outcomes in delayed breast reconstruction. Plast Reconstr Surg Glob Open 5: e1447, 2017.

2. Li M, Chen K, Liu F, Su F, Li S and Zhu L: Nipple sparing mastectomy in breast cancer patients and long-term survival outcomes: An analysis of the SEER database. PLoS One 12: e0183448, 2017.

3. Gunnarsson GL, Bille C, Reitsma LC, Wamberg P and Thomsen JB: Prophylactic nipple-sparing mastectomy and direct-to-implant reconstruction of the large and ptotic breast: Is preshaping of the challenging breast a key to success? Plast Reconstr Surg 140: 449-454, 2017.

4. Zaha $\mathrm{H}$, Inamine $\mathrm{S}$, Naito $\mathrm{T}$ and Nomura $\mathrm{H}$ : Laparoscopically harvested omental flap for immediate breast reconstruction. Am J Surg 192: 556-558, 2006.

5. van Alphen TC, Fechner MR, Smit JM, Slooter GD and Broekhuysen CL: The laparoscopically harvested omentum as a free flap for autologous breast reconstruction. Microsurgery 37: $539-545,2017$.

6 . Kiricuta I: The use of the great omentum in the surgery of breast cancer. Presse Med 71: 15-17, 1963 (In French). 
7. Jimenez AG, St Germain P, Sirois M, Hatheway M and Lethbridge R: Free omental flap for skin-sparing breast reconstruction harvested laparoscopically. Plast Reconstr Surg 110: $545-551,2002$

8. Kroman N, Holtveg H, Wohlfahrt J, Jensen MB, Mouridsen HT, Blichert-Toft $\mathrm{M}$ and Melbye M: Effect of breast-conserving therapy versus radical mastectomy on prognosis for young women with breast carcinoma. Cancer 100: 688-693, 2004.

9. Piper M, Peled AW and Sbitany H: Oncoplastic breast surgery: Current strategies. Gland Surg 4: 154-163, 2015.

10. Zhao WX, Wang B, Yan SY and Zhang LY: Strategy of points, lines and layers in needle assisted laparoscope functional modified neck dissection through bilateral breast approach Zhonghua Wai Ke Za Zhi 54: 823-827, 2016 (In Chinese).

11. Moran MS, Schnitt SJ, Giuliano AE, Harris JR, Khan SA, Horton J, Klimberg S, Chavez-MacGregor M, Freedman G, Houssami N, et al: Society of Surgical Oncology-American Society for Radiation Oncology consensus guideline on margins for breast-conserving surgery with whole-breast irradiation in stages I and II invasive breast cancer. Int J Radiat Oncol Biol Phys 88: 553-564, 2014.

12. Buchholz TA, Somerfield MR, Griggs JJ,El-Eid S, Hammond ME, Lyman GH, Mason G and Newman LA: Margins for breastconserving surgery with whole-breast irradiation in stage I and II invasive breast cancer: American Society of Clinical Oncology endorsement of the Society of Surgical Oncology/American Society for Radiation Oncology consensus guideline. J Clin Oncol 32: 1502-1506, 2014.

13. King PM, Blazeby JM, Ewings P, Franks PJ, Longman RJ, Kendrick AH, Kipling RM and Kennedy RH: Randomized clinical trial comparing laparoscopic and open surgery for colorectal cancer within an enhanced recovery programme. Br J Surg 93: 300-308, 2006.

14. Vignali A, Elmore U, Cossu A, Lemma M, Calì B, de Nardi P and Rosati R: Enhanced recovery after surgery (ERAS) pathway vs traditional care in laparoscopic rectal resection: A single-center experience. Tech Coloproctol 20: 559-566, 2016.
15. Ye QF, Niu Y, She XG, Ming YZ, Cheng K, Ma Y and Ren ZH: Pedicled greater omentum flap for preventing bile leak in liver transplantation patients with poor biliary tract conditions. Hepatobiliary Pancreat Dis Int 6: 470-473, 2007.

16. Tukenmez M, Ozden BC, Agcaoglu O, Kecer M, Ozmen V, Muslumanoglu M and Igci A: Videoendoscopic single-port nipple-sparing mastectomy and immediate reconstruction. J Laparoendosc Adv Surg Tech A 24: 77-82, 2014

17. Zhang P, Luo Y, Deng J, Shao G, Han S and Huang Z: Endoscopic axillary lymphadenectomy combined with laparoscopically harvested pedicled omentum for immediate breast reconstruction. Surg Endosc 29: 1376-1383, 2015.

18. Li N, Zheng Z, Li J, Fan J, Wang T, Zhang J, Wang H, Chen J, Lv Y, Yi J, et al: Immediate breast reconstruction with omental flap for luminal breast cancer patients: Ten clinical case reports. Medicine (Baltimore) 96: e7797, 2017.

19. Romanini MV, Vidal C, Godoy J and Morovic CG: Laparoscopically harvested omental flap for breast reconstruction in Poland syndrome. J Plast Reconstr Aesthet Surg 66: e303-e309, 2013.

20. Song XY, Guan DD, Lin H, Dai Y, Zheng XY, Zhu YP and Wang XF: Immediate breast reconstruction using laparoscopically harvested omental flap after breast-conserving surgery. Zhonghua Zheng Xing Wai Ke Za Zhi 27: 401-405, 2011 (In Chinese)

21. Zaha $\mathrm{H}$ and Inamine S: Laparoscopically harvested omental flap: Results for 96 patients. Surg Endosc 24: 103-107, 2010.

22. Howes BH, Watson DI, Xu C, Fosh B, Canepa M and Dean NR: Quality of life following total mastectomy with and without reconstruction versus breast-conserving surgery for breast cancer: A case-controlled cohort study. J Plast Reconstr Aesthet Surg 69: 1184-1191, 2016

This work is licensed under a Creative Commons Attribution-NonCommercial-NoDerivatives 4.0 International (CC BY-NC-ND 4.0) License. 